\title{
The Neglect of the Global Surgical Workforce: Experience and Evidence from Uganda
}

\author{
Doruk Ozgediz $\cdot$ Moses Galukande $\cdot$ Jacqueline Mabweijano $\cdot$ \\ Stephen Kijjambu · Cephas Mijumbi - Gerald Dubowitz • \\ Samuel Kaggwa $\cdot$ Samuel Luboga
}

(C) Société Internationale de Chirurgie 2008

\begin{abstract}
Background Africa's health workforce crisis has recently been emphasized by major international organizations. As a part of this discussion, it has become apparent that the workforce required to deliver surgical services has been significantly neglected.

Methods This paper reviews some of the reasons for this relative neglect and emphasizes its importance to health systems and public health. We report the first comprehensive analysis of the surgical workforce in Uganda, identify challenges to workforce development, and evaluate current programs addressing these challenges. This was performed through a literature review, analysis of existing policies to improve surgical access, and pilot retrospective studies of surgical output and workforce in nine rural hospitals.

Results Uganda has a shortage of surgical personnel in comparison to higher income countries, but the precise gap is unknown. The most significant challenges to workforce development include recruitment, training, retention, and
\end{abstract}

D. Ozgediz $(\bowtie)$

Department of Surgery and Global Health Sciences, University of California, San Francisco, 513 Parnassus, HSW 1601, Box 0570, San Francisco, CA 94143, USA

e-mail:dozgediz@hotmail.com

M. Galukande $\cdot$ J. Mabweijano · S. Kijjambu ·

S. Kaggwa $\cdot$ S. Luboga

Department of Surgery, Makerere University,

Mulago Hospital Complex, Kampala, Uganda

C. Mijumbi

Department of Anesthesia, Makerere University,

Mulago Hospital Complex, Kampala, Uganda

G. Dubowitz

Department of Anesthesia, University of California,

San Francisco, CA 94143, USA infrastructure for service delivery. Curricular innovations, international collaborations, and development of research capacity are some of the initiatives underway to overcome these challenges. Several programs and policies are addressing the maldistribution of the surgical workforce in urban areas. These programs include surgical camps, specialist outreach, and decentralization of surgical services. Each has the advantage of improving access to care, but sustainability has been an issue for all of these programs. Initial results from nine hospitals show that surgical output is similar to previous studies and lags far behind estimates in higher-income countries. Task-shifting to non-physician surgical personnel is one possible future alternative.

Conclusions The experience of Uganda is representative of other low-income countries and may provide valuable lessons. Greater attention must be paid to this critical aspect of the global crisis in human resources for health.

\section{Introduction}

Surgical burden of disease and "Global Surgical Workforce"

In recent years, most of the increased funding for health to poor countries has been for infectious diseases, such as HIV, tuberculosis, and malaria [1]. By comparison, surgical conditions have been neglected despite a number of recent studies documenting the relative cost-effectiveness of surgery, even when compared with other priority health interventions, such as antiretroviral therapy for HIV disease [2-5]. Surgical conditions account for an estimated $11 \%$ of the global burden of disease, led by injuries, and followed by malignancies, congenital anomalies, complications of childbirth, and cataracts [2]. Meanwhile, reports of surgical output (i.e., ratios of 
operations/population) are exceedingly low in poor countries $[6,7]$. Shortages of surgical human resources are partially responsible for this low output.

The concept of the "global surgical workforce" has yet to be defined and quantified, and any estimates of its size are incomplete and outdated [8]. For the individual patient, the surgical workforce includes workers with skills to identify and prepare patients for surgical procedures, to perform safe operations with appropriate anesthesia, and to care for these patients after surgery. At a population level, this also requires personnel to develop prevention programs and to implement guidelines for the delivery of surgical services. Although some guidelines exist for the required numbers of physicians and nurses per population, there are no corresponding standards or evaluation tools for the numbers of general surgeons, subspecialist surgeons, anesthetists, theater nurses and staff, and other health care workers essential to the delivery of surgical services in low-income countries.

In high-income countries, most surgery is performed by specialists in surgery and anesthesia. By contrast, in rural areas of low-income countries, most surgical procedures are performed by nonspecialists: general doctors (medical officers), nonphysician clinical officers, and anesthetic officers, with variable nursing assistance. In some countries, the use of paramedical cadres in surgery and anesthesia has been promoted with favorable patient outcomes and cost-effectiveness [7, 9-11].

The Bellagio Essential Surgery Group, which first met in June 2007, charted a path to increase access to surgical services in resource-constrained settings and highlighted the need to measure the scale of unmet need for surgical services and to describe surgical workforce needs in subSaharan Africa [12, 13].

Surgical workforce and training in Uganda

In Uganda, there are approximately 2,200 physicians, or 4-8/ 100,000 people with an annual output of 150 physicians. This absolute number, its composition, and its practical value, however, are all uncertain. Some registered physicians are non-Ugandan, and some of the registered Ugandans have migrated externally; an estimated 300 , or $16 \%$ of the physician workforce, are in high-income countries [14]. Surveys have shown that after compensation, working conditions are of the greatest concern and an impetus to work abroad [15]. Perhaps most importantly, there also is an "internal migration" or "internal brain drain" away from clinical services. In other words, many registered Ugandan physicians have shifted from clinical services to positions with greater administrative or research duties in the public health sector with both domestic and international organizations.

For a population of nearly 30 million people, there are approximately 75 specialist-trained general surgeons in
Uganda. The only postgraduate general surgical training program in Uganda at Makerere University has produced approximately three to five trainees per year, chronically under filling its capacity in recent years (Table 1). Some trainees are from Rwanda, Kenya, Tanzania, Malawi, Ethiopia, the Democratic Republic of Congo, and Sudan. Meanwhile, approximately ten surgeons per year graduate from the program in obstetrics and gynecology, and the postgraduate program in anesthesia produces approximately one to three specialists in anesthesia every year. There are approximately ten consultant anesthesiologists for the country and 350 anesthetists. Ten anesthesia physicians have migrated to neighboring countries during the last several years.

In the surgical subspecialties, there are three pediatric surgeons, six neurosurgeons, three plastic surgeons, and three urologists who have all obtained various levels of specialized nonstandardized clinical training abroad [16]. Very little data exist on the supply of subspecialists in sub-Saharan Africa. For most of sub-Saharan Africa, one recent assessment is of approximately 39 pediatric surgeons for the region; in another assessment, there were no neurosurgeons in a group of countries totaling 50 million people $[17,18]$. These ratios are exponentially less than those reported for high-income countries.

There also is a nursing shortage in Uganda, with 16,000 total nurses, or 0.61 per 100,000 people. Compared with other subSaharan African countries with greater external migration, in Uganda this is only $<0.01 \%$ of the nursing workforce [14]. On the surgical wards at Mulago Hospital, the 1,500 bed national referral hospital, there is an average nurse:patient ratio of 1:40. This places much of the burden of care on patient's families, and the full impact of this burden has not been adequately assessed.

For the aforementioned components of the surgical workforce-general and subspecialty surgeons, anesthesiologists, and nurses-no global guidelines exist. As a result, the precise staffing gap for Uganda is unknown. There is still great debate over exactly how this workforce should be developed-whether it should occur through greater specialist or generalist training in surgery [19].

Barriers to recruitment, training, and retention of surgeons and anesthetists

Recent surveys of trainees and professionals in Uganda have emphasized the complex barriers to recruiting, training, and

Table 1 Postgraduates completing selected surgery and anesthesia programs at Makerere University, Kampala, Uganda

\begin{tabular}{lrllll}
\hline & 2003 & 2004 & 2005 & 2006 & 2007 \\
\hline Surgery & 4 & 2 & 12 & 8 & 8 \\
OB-GYN & 14 & 6 & 13 & 3 & 2 \\
Anesthesia & 1 & 3 & 1 & 2 & 1 \\
\hline
\end{tabular}


retaining the workforce for anesthesia and surgery [20, 21]. Perhaps the greatest barrier identified is the relative attractiveness of career paths in public health, pediatrics, medicine, and obstetrics, and gynecology. This is fueled especially by generous international support for research and training collaborations in these fields focused on infectious diseases. Others have raised concerns about the impact of heavy donor support for these vertical programs on the maintenance of essential health services [22].

Other barriers include the comparable length of surgical and anesthesia training and the poor infrastructure for delivery of services. This poor infrastructure compromises quality of care and leads to demoralization of health care providers. Remuneration for government workers is low, and as a consequence specialists engage in private practice to make ends meet. This depletes energy and leaves less time for teaching. For students and medical officers interested in specialty surgical and anesthesia training, there are comparably fewer scholarships available to support postgraduate training. Finally, occupational risks of blood-born infections to health personnel in perioperative care are deterrents to these careers; the risks are comparably greater in these clinical fields $[23,24]$.

\section{Solutions}

A number of educational strategies have been developed to address some of these challenges. Early identification of students interested in perioperative disciplines is underway, with faculty-initiated sponsorship of postgraduate fees for these students who are interested in further training. In addition, curricular innovations have been made in undergraduate surgical education [25]. During the first several years of medical school, students now have earlier clinical exposure through a curricular innovation called "Community-based education and services (COBES)". This provides a more representative community-based and hands-on clinical clerkship experience than that available at the national hospital in the past. Through early exposure, this strategy is designed to increase the number of graduates willing to serve in rural areas after graduation.

For postgraduate trainees, there is an ongoing search for more sources of support for sponsorship because most potential trainees cannot afford the cost. In addition, given the workload of postgraduate training, a service stipend for trainees is being considered. This is currently being piloted by the Ministry of Health in certain fields with especially low numbers, such as anesthesia, but resources are limited.

Also, for postgraduate trainees, the College of Surgeons of East, Central and Southern Africa (COSECSA) has identified several hospitals with dedicated surgeons in each of the eight member countries where some of the professional training will be performed, away from the very busy but poorly funded and equipped tertiary government hospitals, such as Mulago Hospital.

A more comprehensive research program is being developed to investigate the burden of surgical diseases, delivery of services, and unmet need. In Uganda, there has been a relative paucity of surgical research compared with other disciplines. In particular, there is an effort to integrate research on surgical aspects of care of the HIV-infected patient, which has attracted generous support in other fields. Collaborations with other universities are underway as part of this process.

Policies to overcome maldistribution of surgical workforce

In addition to the absolute shortage of health care providers for surgical and anesthetic care, there is an imbalance in distribution, with more than $90 \%$ of physician providers concentrated in Kampala. This creates extraordinary access challenges for the country's $90 \%$ rural population. Surgical services in the periphery are primarily provided by medical officers and anesthetic officers. Several of the strategies developed to overcome these limitations are summarized in Table 2:

1) "Surgical Camps" are conducted once to twice per year for 1-2 weeks at a time by consultant surgeons from all over Uganda to designated regions, under the auspices of the Association of Surgeons of Uganda (ASOU). After sensitizing the community for several weeks before the camps, hundreds of operations are completed during the camps at several facilities. Local medical officers, clinical officers, and health care personnel receive additional training and education during the camps.

According to data from the ASOU, from 1997 to 2004, 13 camps were conducted with 100-900 operations/camp, with an average of 360 operations/camp [26]. The most common operations performed at one of the camps surveyed were inguinal hernias and lipoma excision in both men and women, with gynecological procedures (myomectomy/hysterectomy for fibroids) and thyroidectomies in women, and urological procedures (prostatectomy and urethroplasty) in men. The camps help patients because many of the operations are performed on ambulatory patients near their homes, so there is no referral to higher health units, and this is more affordable to families. In addition, many operations can be done in a short period of time, and local health personnel also receive supplemental 
Table 2 Policies to improve rural access to surgical services in Uganda

\begin{tabular}{lll}
\hline & Advantages & Disadvantages \\
\hline Surgical camps & Large number of cases & Infrequent \\
& Education for local health workers & Limited follow-up \\
& Convenient and affordable for rural poor & Can exhaust local resources \\
Specialist outreach & Access for subspecialty surgery & Limited ability to build capacity due to specialized \\
& & operations \\
& & Limited follow-up in case of complications \\
Surgery at the subdistrict & Improves access for patients & Infrequent \\
& Builds capacity for emergency surgery in local facility & Difficult to support surgery with limited infrastructure \\
& & Recruitment and training of surgical-anesthesia personnel
\end{tabular}

training and supervision. However, they can overwhelm supplies and are very infrequent in any given region.

2) Specialist outreach is periodically conducted regionally by consultant subspecialist surgeons from the national hospital, or from international nongovernmental organizations in orthopedics, plastic surgery, and ophthalmology. Surgical camps and these outreach programs from the national hospital are dependent on funding from the Ministry of Health, which limits their frequency. Studies have shown that outreach can be an effective policy to improve access to specialist care in rural areas, where referral is practically a "myth" [27].

In Uganda, there is a lack of data on detailed output, short- and long-term outcomes, and an integrated evaluation of these programs with the resulting unmet surgical need. For patients with specialized problems, the outreach programs do improve access to care, but fewer patients are treated and the surgical problems can be too specialized for effective hands-on training of local personnel. In addition, it can be difficult for local personnel to treat complications of operations, which may arise due to improper patient selection after the visiting team has left.

3) To better meet rural surgical needs, the Ministry of Health embarked on an ambitious policy several years ago to build and staff operating theaters at the level of the "health subdistrict" or health center IV [28]. Most of these health centers serve a population of 250,000500,000. The primary impetus for this policy was improvement in access to emergency obstetric care. The operations in these theaters were to be performed by a medical officer, and anesthesia to be provided by anesthetic assistants. An 18-month training program was developed to train anesthetic assistants to staff these facilities.

There have been difficulties, however, in recruitment and retention of medical officers and other health personnel to these facilities: incentives for rural service are limited as are resources for support and supervision of the surgical personnel in these settings. Furthermore, medical officers working at these facilities struggle to balance surgical with other clinical and management responsibilities. Infrastructural challenges, such as electricity and running water, also have limited the functionality of these facilities. In a 2004 2005 review of approximately $50 \%$ of the facilities, only $20 \%$ were operational [29]. For the centers with functional operation theaters, their output, the outcomes of care, and the cost-effectiveness of providing surgical services at this further decentralized level of the health care system need to be evaluated.

Evaluation of surgical workforce and output in peripheral hospitals

To better assess surgical output and workforce in peripheral health facilities, retrospective studies have been piloted at nine hospitals in Uganda. Facilities were chosen for regional representation and public/private-not-for profit mix, covering approximately $20 \%$ of the country's population (see map Fig. 1). Four of these hospitals were government-funded district/general hospitals; four were government funded regional referral hospitals; and one was a private-not-for-profit general hospital. The district/general hospitals are designed to serve populations of 100,000 to one million, whereas the regional hospitals are designed to serve populations of one to two million people. All hospitals in the study provide the primary surgical services for these populations, with the exception of Gulu Regional 


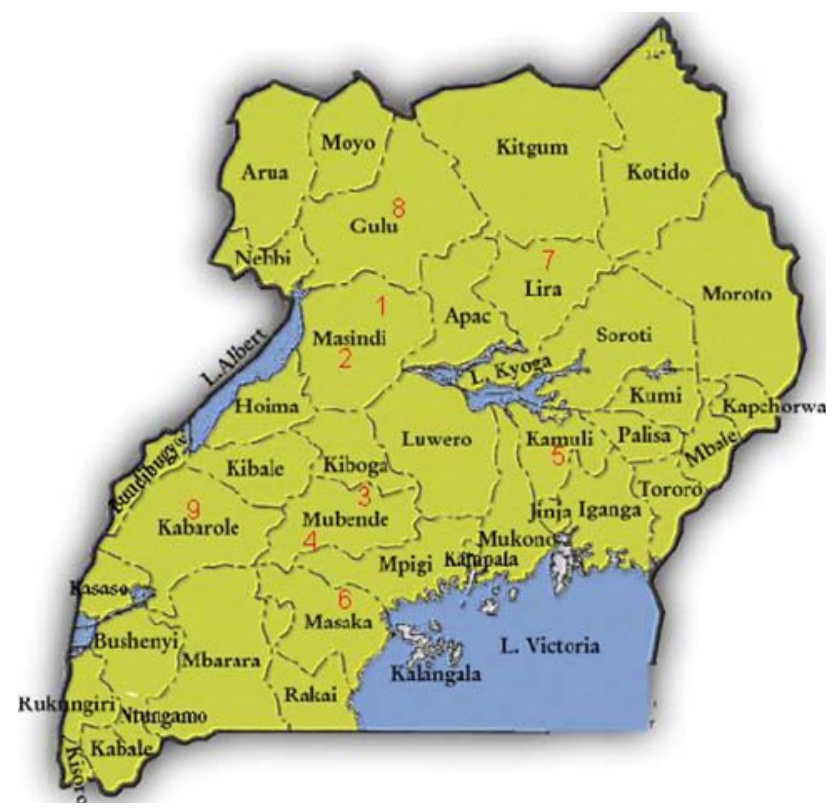

Fig. 1 Map of selected health facilities in Uganda. See Table 3 for corresponding name of health facilities (adapted from www.fao.org/ag/AGP/AGPC/doc/Counprof/ugmap2.gif)

Referral Hospital, where another private hospital in the region also provides specialty surgical services.

Catchment populations were derived from population census and Ministry of Health. In each hospital, the surgical workload was reviewed using data from April 2006 to March 2007 recorded in operative log books and outpatient procedure records. Operations were subsequently classified by general surgery and obstetric-gynecologic operations. The number and classification of selected surgical personnel also was assessed on site.

Hospitals varied evenly by the majority of general surgery or OB-GYN operations (Table 3). Overall, the most common general surgical operations performed in the public district hospitals were surgical toilet and suture (generally a washout and closure of a wound), incision and drainage, herniorrhaphy, and laparotomy (Table 4). Meanwhile, the most common OB-GYN procedures were cesarean section, tubal ligation, salpingectomy, and evacuation (Table 5). Although previous reports have classified operations as major or minor, this was not undertaken in this study because of an inability to delineate the duration and complexity of procedures from operative logbooks alone.

The operation rates/catchment population ranged from 86 to $622 / 100,000$, with the lowest rate at the regional hospital in Gulu, where as previously stated, there is another private regional hospital in the region providing surgical services. Rates are consistent with previous estimates from Uganda, and the region where surgical output is far behind those in higher-income countries [6, 30-32]. This is despite the fact that these estimates are dated by 10 20 years and raises the question of whether any progress has been made during this time to improve access to surgical care for rural populations.

The data also confirm that the general hospitals have no specialist general or OB-GYN surgeons, with the exception of one obstetrician at Mityana Hospital (Table 6). All operations in these facilities are performed by medical officers, and anesthesia is provided by anesthetic officers. Although anesthesia staffing was not formally collected for this survey, it is known that there are no anesthesia-physicians in the general hospitals.

These preliminary results reveal a paucity of some operations, such as appendectomies and breast operations, in rural facilities. It is unclear whether these patients never reach the hospital, whether they are referred to other facilities, or whether there is a regionally diverse epidemiology of surgical disease with fewer cases. Alternatively, providers may not be available to perform operations due to competing

Table 3 Surgical output at selected hospitals in Uganda, April 2006-March 2007

\begin{tabular}{lllrrrrrr}
\hline $\begin{array}{l}\text { Hospital } \\
\text { number }\end{array}$ & Hospital & Type & $\begin{array}{l}\text { Catchment } \\
\text { population }\end{array}$ & Beds & $\begin{array}{l}\text { OB-GYN } \\
\text { operations }\end{array}$ & \multicolumn{2}{l}{$\begin{array}{l}\text { General surgery } \\
\text { operations }\end{array}$} & $\begin{array}{l}\text { Total } \\
\text { output }\end{array}$ \\
\hline 1 & Kiryadongo & PG & 125,000 & 100 & $46(17 \%)$ & $219(83 \%)$ & 265 & 212 \\
2 & Masindi & PG & 125,000 & 100 & $338(54 \%)$ & $284(46 \%)$ & 622 & 498 \\
3 & Mubende & PG & 250,000 & 100 & $1071(69 \%)$ & $483(31 \%)$ & 1554 & 622 \\
4 & Mityana & PG & 250,000 & 100 & $661(56 \%)$ & $519(44 \%)$ & 1180 & 472 \\
5 & Kamuli & PNFPG & 500,000 & 153 & $736(41 \%)$ & $1046(59 \%)$ & 1782 & 356 \\
6 & Masaka & PRRH & 850,000 & 330 & $933(53 \%)$ & $822(47 \%)$ & 1755 & 206 \\
7 & Lira & PRRH & 500,000 & 254 & $467(46 \%)$ & $547(64 \%)$ & 1014 & 203 \\
8 & Gulu & PRRH & $1,200,000$ & 367 & $413(40 \%)$ & $616(60 \%)$ & 1029 & 86 \\
9 & Fort Portal & PRRH & 500,000 & 400 & $504(54 \%)$ & $427(46 \%)$ & 931 & 186 \\
\hline
\end{tabular}

Hospital number-Corresponds to map

Hospital type-PG, public general (or district); PNFPG, private not-for-profit general; PRRH, public regional referral hospital

Ratio, total operations $/ 100,000$ population 
Table 4 General surgery operations at selected hospitals

\begin{tabular}{lccccc}
\hline Hospital & General surgery & Surgical toilet suture & Incision + drainage & Hernia repair & Laparotomy \\
\hline Kiryadongo & 219 & $66(30 \%)$ & $56(26 \%)$ & $13(6 \%)$ & $4(2 \%)$ \\
Masindi & 284 & $52(18 \%)$ & $38(13 \%)$ & $86(34 \%)$ & $78(16 \%)$ \\
Mubende & 483 & $125(26 \%)$ & $75(15 \%)$ & $188(36 \%)$ & $13(15 \%)$ \\
Mityana & 519 & $179(35 \%)$ & $202(19 \%)$ & $100(10 \%)$ & $13(3 \%)$ \\
Kamuli & 1046 & $277(27 \%)$ & $37(13 \%)$ & $45(8 \%)$ & $13 \%)$ \\
Masaka & 822 & $50(6 \%)$ & $54(8 \%)$ & $112(17 \%)$ \\
Lira & 547 & $64(12 \%)$ & $97(23 \%)$ & $65(15 \%)$ \\
Gulu & 616 & $104(16 \%)$ & $63(15 \%)$ & & $30(5 \%)$ \\
Fort Portal & 427 & & $51(12 \%)$ \\
\hline
\end{tabular}

Table 5 Obstetric operations at selected hospitals

\begin{tabular}{lrllclll}
\hline Hospital & Total & Cesarean section & Bilateral tubal ligation & Salpingectomy & Hysterectomy & Evacuation & Dilation and curettage \\
\hline Kiryadongo & 46 & $26(57 \%)$ & $5(11 \%)$ & $1(2 \%)$ & $1(2 \%)$ & 0 & 0 \\
Masindi & 338 & $162(48 \%)$ & $29(9 \%)$ & $18(5 \%)$ & $12(4 \%)$ & $98(29 \%)$ & $1(0.2 \%)$ \\
Mubende & 933 & $522(49 \%)$ & $35(33 \%)$ & $5(0.5 \%)$ & $41(4 \%)$ & $354(38 \%)$ & $47(5 \%)$ \\
Mityana & 661 & $475(72 \%)$ & $77(12 \%)$ & $18(3 \%)$ & $21(3 \%)$ & $141(21 \%)$ & $4(0.6 \%)$ \\
Kamuli & 736 & $478(65 \%)$ & $65(9 \%)$ & $65(9 \%)$ & $71(10 \%)$ & 0 & $55(8 \%)$ \\
Masaka & 933 & $831(89 \%)$ & $36(4 \%)$ & $16(2 \%)$ & $19(2 \%)$ & $2(0.2 \%)$ & 0 \\
Lira & 467 & $400(85 \%)$ & 0 & $13(3 \%)$ & 0 & $13(3 \%)$ & 0 \\
Gulu & 413 & $162(39 \%)$ & 0 & $17(4 \%)$ & $23(5 \%)$ & $28(7 \%)$ & $19(0.5 \%)$ \\
Fort Portal & 504 & $411(82 \%)$ & $10(2 \%)$ & $7(2 \%)$ & $31(7 \%)$ & $1(0.2 \%)$ & $3(0.6 \%)$ \\
\hline
\end{tabular}

responsibilities or inadequate infrastructure, or procedures may be misclassified. For example, "surgical toilet and suture" could mean anything from sequestrectomy for osteomyelitis, washout of an open fracture, tendon injury, washing of a burn wound, or simple laceration. Similarly, laparotomies may be performed for trauma, infection, perforated viscus or appendicitis, or bowel obstruction. The application of splints for fractures and other index orthopedic procedures also were scantly recorded at facilities.

Table 6 Staffing at selected hospitals, 2006

\begin{tabular}{lllc}
\hline Hospital & $\begin{array}{l}\text { General } \\
\text { surgeon }\end{array}$ & $\begin{array}{l}\text { OB-GYN } \\
\text { surgeon }\end{array}$ & $\begin{array}{l}\text { Medical } \\
\text { officer }\end{array}$ \\
\hline Kiryadongo & 0 & 0 & 3 \\
Masindi & 0 & 0 & 2 \\
Mubende & 0 & 0 & 2 \\
Mityana & 0 & 1 & 4 \\
Kamuli & 0 & 0 & 3 \\
Masaka & 2 & 1 & 6 \\
Lira & 1 & 2 & 4 \\
Gulu & 1 & 3 & 12 \\
Fort Portal & 2 & 2 & 1 \\
\hline
\end{tabular}

The reasons why there are higher rates of surgery in all general hospitals over regional hospitals have been previously reported but must be further investigated [33]. One might expect that more complex procedures, such as laparotomies and hysterectomies, are performed at regional hospitals as specialists are available; however, this is not supported by the composition of procedures available. Surgical output in the regional hospitals is limited by their poor infrastructure and by the fact that the specialist surgeons work at private facilities and are overburdened with administrative duties in addition to supervising the district hospitals.

In addition, there was no classification of operations as emergent or elective. This information would be useful from a planning perspective. A great proportion of the obstetric operations, especially caesarian sections, were likely emergencies, but little is known about the urgency of the general surgery operations by comparison. For elective procedures, referral patterns also are not recorded. For some facilities, the catchment area must be better defined, because smaller facilities may be providing surgical services, such as some of the newly equipped health center IVs.

Plans for further hospital assessment are being developed based on this pilot data. There is a need to determine 
exactly who is performing each operation at facilities where there are both specialists and general physicians or clinical officers with surgical skills. Focus group discussions with surgical personnel and more detailed workforce assessments are in process to plan training, education, and improvements in infrastructure. There is a complete lack of prospective data recording diagnoses, procedures, and outcomes except for facility logbooks. Currently, a template is being developed with the WHO and international collaborators to standardize data collection and to prospectively assess outcomes and cost-effectiveness of surgical care. This data will be used to further analyze variation in surgical output between hospitals and to design training programs for surgical staff. The facility-based data will be compared with community-based surveys to determine more precisely the level of unmet need. The need to train paramedical cadres, which has been successful in other countries, may subsequently need to be considered in Uganda to meet unmet need [34].

\section{Conclusions}

Within the discussion on human resources for health to date, the emphasis on the global surgical workforce has been poorly developed. Compared to the relative advocacy for other diseases, there seems to be a tacit global acceptance of the "surgical status quo," which is apparent to clinicians providing surgical care in poor countries: many patients with treatable surgical problems present at late stages of disease, live with a chronic surgical disability, or die from a lack of access to care [35]. The surgical burden of disease is significant, and there is a global shortage of surgical manpower, which has been inadequately delineated. In Uganda, there are relatively few specialist surgeons and anesthesiologists, and there is a difficulty in recruiting and retaining surgeons. Curricular innovations, international collaborations, and attempts to build research capacity are all attempting to face these challenges. Surgical camps, specialist outreach, and construction of theaters at the subdistrict level have all attempted to improve access to surgical care for rural populations. These programs have all experienced significant challenges, especially in sustainability. Surveys from nine hospitals show a near even distribution of general surgical and obstetric cases, and surgical output is low, consistent with previous estimates. Public and private general/district hospitals are primarily staffed by medical officers and anesthetic officers and have higher productivity ratios than regional hospitals for unclear reasons. The existing data collection system for surgery at the district levels (i.e., operative logbooks) is inadequate to perform robust comparisons across facilities. Further research in these areas, along with studies of unmet need for specific conditions, is underway in Uganda. At a global level, there is a need to develop data collection tools and guidelines for surgical services to improve public health.

\section{References}

1. Garrett L (2007) The Challenge of Global Health. Foreign Affairs, Jan-Feb

2. Debas H, Gosselin R, McCord C, Thind A (2006) Surgery. In: Jamison D (ed) Disease control priorities in developing countries, 2nd edn. World Bank, Washington DC

3. Gosselin RA, Thind A, Bellardinelli A (2006) Cost/DALY averted in a small hospital in Sierra Leone: what is the relative contribution of different services? World J Surg 30:505-511

4. McCord C, Chowdhury Q (2003) A cost-effective small hospital in Bangladesh: what it can mean for emergency obstetric care. Int J Gynaecol Obstet 81:83-92

5. Laxminarayan R, Mills AJ, Breman JG, Measham AR, Alleyne G, Claeson M et al (2006) Advancement of global health: key messages from the Disease Control Priorities Project. Lancet 367(9517):1193-1208

6. Nordberg E, Holmberg S, Kiugu S (1995) Output of major surgery in developing countries. Towards a quantitative evaluation and planning tool. Trop Geogr Med 47:206-211

7. Fenton PM (1997) The epidemiology of district surgery in Malawi: a two-year study of surgical rates and indices in rural Africa. East Cent Afr J Surg 3:33-41

8. MacGowan WA (1987) Surgical manpower worldwide. Bull Am Coll Surg 72:5-9

9. Vaz F, Bergstrom S, Vaz ML, Langa J, Bugalho A (1999) Training medical assistants for surgery. Bull World Health Organ 77:688-691

10. Kruk M, Pereira C, Vaz F, Bergstrom S, Galea S (2007) Economic evaluation of surgically trained assistant medical officers in performing major obstetric surgery in Mozambique. BJOG 114:1253-1260

11. Longombe AO (1997) Surgical training of nurses for rural areas: necessity or aberration? East Cent Afr J Surg 3:43-47

12. The Bellagio Essential Surgery Group (2007) Increasing access to surgical services in resource-constrained settings in sub-Saharan Africa [in preparation]). Bellagio Essential Surgery Group, Italy

13. Bellagio Essential Surgery Group (2007) Improving Access to Surgery in sub-Saharan Africa. Available at: http://www. globalhealthsciences.ucsf.edu/bulletins/bulletins.aspx. Accessed 1 Oct 2007

14. WHO (2006) World Health Report: working together for health

15. Matsiko C, Kiwanuka J (2003) Overview of Human Resource for Health in Uganda. Health Policy and Development

16. Department of Surgery, Makerere University and Association of Surgeons of Uganda, 2007

17. Bickler SW, Rode H (2002) Surgical services for children in developing countries. Bull World Health Organ 80:829-835

18. El Khamlichi A (2005) Neurosurgery in Africa. Clin Neurosurg 52:214-217

19. Loefler IJ (2002) The specialist-generalist controversy. S Afr J Surg 40:87-89

20. Galukande M, Kijjambu S, Luboga S (2006) Improving recruitment of surgical trainees and training of surgeons in Uganda. East Cent Afr J Surg 11:17-24

21. Hodges SC, Mijumbi C, Okello M, McCormick BM, Walker IA, Wilson IH (2007) Anesthesia services in developing countries: defining the problems. Anesthesia 62:4-11 
22. Angemi D, Oyugi J, Aziz I, Kyamukama T (2007) The money flows, the boy dies. International Herald Tribune, 25 April

23. Nsubuga FM, Jaakkola MS (2005) Needle stick injuries among nurses in sub-Saharan Africa. Trop Med Int Health 10:773-781

24. Newsom DH, Kiwanuka JP (2002) Needle-stick injuries in a Ugandan teaching hospital. Ann Trop Med Parasitol 96:517-522

25. Kiguli-Malwadde E, Kijjambu S, Kiguli S, Galukande M, Mwanika A, Luboga S et al (2006) Problem Based Learning, curriculum development and change process at Faculty of Medicine, Makerere University, Uganda. Afr Health Sci 6:127-130

26. Uyeno L, Watya S (2005) Strategies for improving surgical access in rural Uganda: evaluation of multifaceted outreach and specialist camps; presentation

27. Gruen RL, Bailie RS, Wang Z, Heard S, O'Rourke IC (2006) Specialist outreach to isolated and disadvantaged communities: a population-based study. Lancet 368(9530):130-138

28. Health subdistricts in Uganda. Ministry of Health, Kampala, 1999
29. Annual health sector performance report. Ministry of Health, Kampala, 2006

30. Watters DA, Bayley AC (1987) Training doctors and surgeons to meet the surgical needs of Africa. Br Med J (Clin Res Ed) 295(6601):761-763

31. Nabembezi JS, Nordberg E (2001) Surgical output in Kibaale district, Uganda. East Afr Med J 78:379-381

32. Levy LF, Chigwanda PC, Mauchaza B, Hatfill S, Bakasa R (1986) Prevalence of untreated disease in rural Zimbabwe. Cent Afr J Med 32:159-163

33. King M, Bewes P, Cairns J, Thornton J (1990) Primary surgery. Oxford University Press, Oxford

34. Saswata B, Omar F, Aubery RJ, Jaffer B, Walsh M (2005) Bridging the health gap in Uganda: the surgical role of the clinical officer. Afr Health Sci 5:86-89

35. Nundy S (1999) Difficulties of surgery in the developing world: a personal view. Lancet 353(Suppl 1):S121-S123 